\title{
Contribution à l'étude quantitative des protéines sériques du zébu arabe du Tchad
}

\author{
por R. QUEVAL
}

Dans le cadre des études menées dans le service de virologie du Laboratoire central de recherches vétérinaires de Farcha, au Tchad nous avons eu la possibilité d'étudier ies limites de variations des protéines sériques chez de jeunes zébus de race arabe dans des conditions physiologiques normales par la méthode de iélectrophorèse sur papier. Ce sont les résultats obtenus qui font l'objet de la présente note.

\section{TRAVAUX ANTERIEURS}

L'étude des protéines sériques des bovidés n'a jusqu'ici fait l'objet que de quelques recherches dont il convient de rappeler ci-après les plus marquantes. Les publications de anque allemande rapportent les travaux de Stockl et Zacherl (1) puis de Stockl (2) qui ont étudié les animaux dans leur état normal puis après immunisation et en particulier ont fixé les 'imites de variations des gamma globulines au cours de l'immunisation.

Boguth (3). Chopard (4), et Winkler (5) ont déterminé les protéines sériques des bovins avec des considérations sur l'influence de divers facteurs (sexe, race, âge, état de gestation, travail et alimentation).

Boguth (6), Geinitz (7). Harturig (8) et Weber (9) après avoir fixé les variations des protéines sériques des animaux dans des conditions physiologiques normales étudient les modifications électrophorétiques quantitatives et qualitatives dans divers états pathologiques ou chez les animaux producteurs de sérums antitoxiques ou antibactériens.
En Italie, Montemagno et Agresti (10) ont contribué à l'étude quantitative des protéines, giycoprotáines et lipoprotéines des bovidés, ainsi que des auteurs anglo-saxons: Badish, Henderson et Brookly (11). Campbell (12) et Rooney (13).

Jones (14) d'une part et Garner (15) d'autre part ont étudié par des méthodes biochimiques ies variations du taux des protéines sériques chez 'es zébus.

Enfin, les publications de langue française sont représentées par les thèses de Panisset (16) Vermorel (17) et Kuentz (18).

\section{MATERIELS ET METHODES}

Notre étude a porté sur 60 : bouvillons zébus arabes, de sexe mâle, âgés de 8 à 15 mois ne présentant pas de signes cliniquement décelables de maladies aiquës ou chroniques.

Les práièvements de sang furent. effectués stérilement par ponction intraveineuse au niveau de la veine jugulaire. Sitôt après coagulation les échantilions de sang furent immédiatement et soigneusement centrifugés. Les sérums non exáminés dans les quelques heures qui suivirent le prélèvement furent conservés congelés à - $18^{\circ} \mathrm{C}$. La stérilité des manipulations et la rapidité des opérations évitent les modifications que peuvent subir les diverses fractions sériques pár protéolyse ou hémolyse.

L'appareil utilisé dans cette étude est un appare:í à électrophorèse sur papier construit par les Etablissements Jouan qui permet l'enregistrement automatique de la courbe directement

Reçu pour publicatiss : juiciet 1959. 
en densité optique et de plus est équipé d'un intégrateur de courbes.

Les constantes techniques des expériences furent les suivantes; tension : 400 volts, durée de l'électrophorèse : 3 heures 30 minutes; tampon véronal de $\mathrm{pH} 8,8$ et de force ionique 0,05.

La séparation électrophorétique, lerminée, les bandes de papier Arches 304 sont séchées au moyen d'un sécheur infra-rouge (Jouan) à une température de $70^{\circ} \mathrm{C}$ pendant 30 minutes. La rapidité du séchage ef la position horizontale du papier permettent une bonne fixation et l'obtention de taches régulières.

Les protéines sériques sont révélées suivant la méthode classique au bleu de bromophénol, cependant nous ajoutons selon les indications de $G$. Sandor et Mlle Y. Sabetay (19) à la solution de bleu de bromophénol saturée de sublimé, 10 p. 100 de son volume d'une solution de bleu de bromothymol à I p. 100. Pour avoir des conditions de coloration identiques toutes les bandes sont colorées en même temps par immersion pendant 10-15 minutes. Les bandes saturées de colorant sont ensuite plongées dans des cuves contenant de l'eau naturelle, celle-ci est renouvelée toutes les 30 minutes pendant 3 heures. Par cette technique de coloration les taches deviennent sensiblement plus foncées alors que le fond du papier est totalement décoloré.

Les bandes colorées et séchées sont passées au photomètre; le dispositif d'enregistrement semi-automatique et la table d'intégration de l'appareil permettent d'obtenir simultanément le tracé de la courbe d'absorption et l'intégration de cette courbe.

\section{RESULTATS}

Les résultats obtenus sont résumés et colligés dans le tableau I:

TABLEAU : I

\begin{tabular}{|c|c|c|c|c|c|}
\hline Paramètres & Albumines & Alpha & Bêta & Gamma & Rapport A/G \\
\hline Moyenne & 31,1 & 21,1 & 13,3 & 33,7 & 0,45 \\
Ecart type & 5,2 & 3,5 & 3,7 & 6,2 & 0,12 \\
$\begin{array}{c}\text { Test de } \\
\text { Pearson }\end{array}$ & 20,5 & 0,19 & 3,5 & 13,3 & 6,9 \\
\hline
\end{tabular}

Les résultats de cette étude n'ont de valeur que dans la mesure où l'on peut les extrapoler à un vaste ensemble. Bien que les moyens dont nous disposions ne nous aient pas permis d'étudier plus d'un échantillon nous pouvons légitimement procéder à cette extrapolation l'échantillon ayant été tiré au hasard parmi le cheptel de la région du Chari-Baguirmi qui doit compter de 400.000 à 500.000 têtes dont environ 80.000 à 100.000 animaux sont âgés de $\mathrm{I}$ à 2 ans.

Le tableau II rapporte les estimations de la moyenne et de l'écart type de l'ensemble pour des intervalles de confiance à 0,95 .

TABLEAU II

\begin{tabular}{|c|c|c|c|c|c|}
\hline Paramètres & Albumines & Alpha & Bôta & Gamma & Rapport A/G \\
\hline \multirow{3}{*}{ Moyenne } & 29,8 & 20,1 & 12,3 & 32,1 & 0,41 \\
& 32,5 & 22,0 & 14,3 & 35,3 & 0,49 \\
& 2,7 & 1,8 & 1,7 & 3,0 & 0,3 \\
Ecart type & 4,0 & 2,6 & 2,5 & 4,3 & 0,4 \\
\hline
\end{tabular}

\section{DISCUSSION}

De toutes les études électrophorétiques, la diversité des résultats obtenus fait ressortir qu'ils ne peuvent être considérés comme établissant des normes pour les bovins en général, les différents auteurs parvenant à des résultats similaires en ce qui concerne certaines fractions mais par contre de flagrantes divergences apparaissant pour d'autres. Il est évident que ces discordances sont liées d'une part à la diversité des techniques électrophorétiques employées ef d'autre part à des différences de conditions. expérimentales et de matériel d'étude.

Nos observations ont été faites chez de jeunes zébus, dans une population homogène subissant des conditions identiques d'environnement (alimentation, climat) et par voie de conséquence nos resultats ne sont valables et adaptables qu'à l'ensemble du cheptel de la région du Chari-Baguirmi pour des zébus de même âge, de même race, placés dans des conditions identiques.

L'étude électrophorétique des protéines du «Zébu » comparée à celle des « Bovins 》 permet de constater que les valeurs moyennes 
obłenues présentent des différences marquées. Ce parallèle met en évidence chez le zébu une hypoalbuminémie constante et fortement prononcée et par voie de conséquence une augmentation du taux des globulines sériques d'où un rapport Albumines/Globulines $(A / G)$ très faible.

Cette dysprotéinémie peut être attribuée à des affections fréquentes en pathologie tropicale intéressant les systèmes hépatique ou extrahépatique ou le système réticulo-endothélial et pouvant par là modifier l'équilibre protidique de telle sorte que l'on puisse considérer la diminution de l'albumine sérique secondaire à l'augmentation des globulines qui serait le phénomène primitif, essentiel; cependant il n'est pas inutile de mentionner les théories selon lesqueiles l'albumine ou un produit de dégradation métabolique de l'albumine serait à l'origine des globulines. De plus l'élaboration des anticorps sériques antitoxiques ou antimicrobiens a pour support les gamma globulines et que nombre d'in. fections chroniques lanaplasmoses, piroplasmoses, théilérioses) déterminent une hyperactivité des tissus lymphoides formateurs d'anticorps et par suite la libération des gamma giobuiines.

La dysprotéinémie peut encore être liée soit à un état de malnutrition, soit à des séquelles d'affections parasitaires chroniques ou aiguës, soit à des différences physiologiques résultant d'une adaptation au milieu tropical.

Les conditions nutritionneiles en particulier peuvent expliquer l'hypoalbuminémie observée: en effet l'albumine en presque tota'ité d'origine hépatique subit soit des variations lors de déficiences protéiniques alimentaires, soit des transformations lors de diverses affections qui retentissent aussi bien sur le système hépatique qu'extra-hépatique et qui se traduisent par des processus de destruction toxique ou de transformation en globulines alpha.

En outre l'augmentation des aipha globulines est liée à toute inflammation ou à toute destruction tissulaire quelle qu'en soit la cause: en particulier les globulines alpha 2 contiennent d'une part l'haptoglobine et d'autre part des glucoprotéides dont le taux s'élève au cours de toutes inflammations; or les maladies dites autoentretenues du bétail dans les pays tropicaux sont constantes.
La légère hyperglobulinémie bêta parfois observée semble résulter du fait que certains anticorps sont des molécules protéiques faisant partie des globulines intermédiaires entre les fractions bêta et gamma et qu'il existe en outre dans le sérum d'autres protéines immunes telles que les isoagglutinines ef les réagines allergiques qui sont associées soit à des globulines alpha 2 , soit à des bêta globulines.

\section{CONCLUSION}

Quoique nos observations non choisies aient porté sur des animaux apparemment en bon état de santé que nous avons considéré comme normaux, on ne doit pas négliger les maladies ou syndrômes qui du point de vue clinique ou étiologique n'ont pas une délimitation ef une autonomie précises; les états précarentiels inapparents, les maladies infectieuses ou parasitaires qui présentent des latences cliniques ou qui créent un équilibre de l'organisme entre l'hôte et le parasite ou bien qui laissent des séquelles, enfin les maladies auto-entretenucs, soit toutes maladies largement répandues sous les climats tropicaux.

\section{BIBLIOGRAPHIE}

1. STOCKL ef ZACHERL (M.K.). - Hoppe Seyler's Zeitschr. Physiol. Chem., 1953, 293, 278.

2. STOCKL (W.). - Wien. tierärstl. Monatschr., $1956,43,150-7 ; 226-40$ et $402-30$.

3. BOGUTH (W.). - Zbl. vet. Med., 1953, I, 168.

4. CHOPARD (P.). - - Schweizer Arch. Tierheilk. 1954, 96, 252-60.

5. WINKLER (D.). - Deutsche tierärztl. Wschr. $1955,62,515-7$.

6. BOGUTH (W.). - Zbl. vet. Med., 1954, I, 311.

7. GEINITZ (W.). - Klin. Wschr., 1954, 32, 1108-11.

8. HARTUNG (J.). - Deutsche tierärztl. Wschr., 1954, 61, 300-1.

9. WEBER (W.). - Schweizer Arch. Tierheilk., 1955, 97, 222-9. 